\title{
Educación remota en contexto de pandemia: caracterización del proceso educativo en las universidades chilenas
}

\author{
Verónica Villarroel ${ }^{1}$, Carlos Pérez², Cristian A. Rojas-Barahona ${ }^{3^{*}, 4}$ y Ricardo García ${ }^{5}$ \\ (1) Centro de Investigación y Mejoramiento de la Educación (CIME), Facultad de Psicología, Universidad del Desarrollo, \\ Ainavillo \#456, Concepción, Chile. (Correo-e: vvillarroel@udd.cl) \\ (2) Instituto de Ciencias Sociales, Universidad de O'Higgins, Avenida Libertador Bernardo O'Higgins \#611, Rancagua, Chile. \\ (Correo-e: carlos.perez@uoh.cl) \\ (3) Facultad de Psicología, Universidad de Talca, Av. Lircay S/N, Talca, Chile. (Correo-e: c.rojas@utalca.cl) \\ (4) Facultad de Ciencias de la Educación, Universidad de Talca, Oriente \#591, Linares, Chile. (Correo-e: c.rojas@utalca.cl) \\ (5) Centro de Desarrollo e innovación de la Docencia (CeDID), Universidad Católica de Temuco, Montt \#56, Temuco, Chile. \\ (Correo-e: rgarcia@uct.cl)
}

* Autor a quien debe ser dirigida la correspondencia

Recibido Mar. 4, 2021; Aceptado May. 5, 2021; Versión final Jul. 2, 2021, Publicado Dic. 2021

\section{Resumen}

El objetivo de este estudio es caracterizar el proceso educativo remoto en Chile durante la pandemia COVID-19 desde la experiencia de docentes y estudiantes. Se consideran aspectos teóricos sobre las mejores prácticas de educación remota, el género, y el área disciplinar. Se aplicaron dos instrumentos (en línea) de respuesta cerrada sobre la percepción de condiciones físicas-tecnológicas, competencias digitales, la relación profesor-estudiantes, el proceso de enseñanza y evaluación, y las emociones en educación remota en pandemia. La muestra está compuesta por un grupo de 1750 estudiantes y 654 profesores de 34 universidades chilenas. Los resultados dan cuenta de elementos transversales, con ciertas diferencias de acuerdo a niveles formativos, al género y al área disciplinar. Se concluye que existe la necesidad de formación pedagógica para docentes, la necesidad de más diálogo profesor-estudiante, y la mejora de las prácticas evaluativas que integran la retroalimentación, el trabajo colaborativo y la incorporación de estrategias de enseñanza diferentes a la educación presencial.

Palabras clave: educación remota; pandemia; enseñanza; aprendizaje; relación estudiante-docente

\section{Distance education in a pandemic context: educational process characterization in Chilean universities}

\begin{abstract}
The main goal of this study is to characterize Chilean distance education processes during the COVID-19 pandemic from the experience of professors and students. Theoretical aspects are assessed in relation to the best-know distance education practices, gender, and field of study. Two online closed-answer instruments are applied to assess perceptions on technological conditions, surrounding environment, digital skills, professorstudent relationships, teaching and evaluation processes, and emotions. The sample consists of 1750 students and 654 professors from 34 Chilean universities. The results show multiple cross-cutting elements that differ according to year of study, student gender, and university program. It is concluded that there is a need for professor training in pedagogy, for reinforcing student-professor dialogue, and for developing improved assessment practices that integrate feedback, collaborative work, and the inclusion of distance education teaching strategies that are different from that of face-to-face education.
\end{abstract}

Keywords: distance education; pandemic; teaching; learning; student- professor relation 


\section{INTRODUCCIÓN}

La digitalización de la sociedad y su impacto en la economía y el aprendizaje comenzó en la década de los 70 . Una de las primeras iniciativas de educación remota fue registrada por la Open University en Inglaterra en 1970, luego, desde 1980, se sumaron otros países europeos (Durán y Estay, 2016). Casi al finalizar la década de los 80, se conocieron las primeras iniciativas latinoamericanas, lideradas por el Tecnológico de Monterrey en México, las que a partir de 1990 se ampliaron a los demás países de la región. Actualmente, más de trece millones de personas alrededor del mundo se encuentran inscritas en cursos virtuales fuera de su país de origen (Manyika et al., 2016). En países desarrollados la educación remota es bastante común; por ejemplo, en Australia y Estados Unidos superan el $20 \%$ de la oferta (Yong et al., 2017); mostrando que la tendencia desde los años 70 ha ido en franca expansión, aunque con diferencias en su desarrollo en las distintas regiones del mundo.

En América Latina en cambio, el crecimiento de la educación remota ha avanzado lentamente, pasando de un 1.3 a un $7.5 \%$ entre los años 2000 al 2012; Brasil destaca con un 17.1\% de matrículas en educación superior en línea (Durán y Estay, 2016). En Chile, la educación remota es aún incipiente: según cifras del Servicio de Información de Educación Superior del Ministerio de Educación de Chile, en el 2018, un poco más de 35 mil estudiantes se matricularon para seguir este tipo de jornada. Aunque esta cifra implica un alza de $140.7 \%$ entre el período 2014-2018, no alcanza a llegar al 5\% de la matricula de educación superior y las universidades más reconocidas en Chile ofertan pregrados casi exclusivamente presenciales (SIES, 2019).

Hasta antes de la contingencia COVID-19, coexistían atendiendo a nichos diferenciados, las llamadas formaciones presenciales, semipresenciales y a distancia, cada una diseñada desde su conceptualización curricular para el ambiente presencial o virtual en el que fuera requerido. Así, la denominada formación a distancia era una modalidad de enseñanza formal y reconocida, a la que en general optaban perfiles más asociados a profesionales en ejercicio laboral, con altas restricciones de tiempo o desplazamiento. También hay que mencionar la existencia previa de los denominados cursos masivos, en línea y en abierto, conocidos por si acrónimo en inglés, "MOOC", que por sus características de gratuidad, flexibilidad horaria y transversalidad, se configuraban como una opción complementaria, aunque con ciertas debilidades como los escasos sistemas de tutorización y evaluación respecto de otros modelos de enseñanza (López-Meneses et al., 2020).

En este contexto de avance progresivo de la educación remota en Chile, la pandemia COVID-19 con sus restricciones de movilidad e interacción física cercana, obligó a las universidades de manera disruptiva a adaptar sus propuestas formativas, principalmente de pregrado, las cuales fueron diseñadas originalmente mediante una visión curricular con una fuerte componente presencial, y muy alineadas con las competencias del perfil de egreso y los estándares de acreditación, debiendo ser impartidas provisoriamente bajo modalidades de educación remota. Este cambio se generó con una rapidez inusual y sin la experiencia requerida, ya que en general, al estar las formaciones de pregrado orientadas bajo un paradigma presencial, en Chile, la mayoría de las universidades no contaban con las plataformas tecnológicas necesarias para este giro formativo hacia una modalidad remota: los docentes no habían sido debidamente capacitados en competencias digitales ni en el desarrollo de estrategias de aprendizaje para los entornos virtuales requeridos; tampoco se habían evaluado las condiciones básicas de sus estudiantes y profesores para enseñar y aprender desde el hogar. Sin embargo, en pocas semanas y muy cerca del inicio del año académico del 2020, se instaló la educación remota en la educación superior chilena. A pesar de las diferencias interculturales, este hecho disruptivo tuvo consecuencias similares en el mundo universitario en general. Según el propio reporte de la Asociación Internacional de Universidades, la pandemia ha impactado "... a menudo de forma dramática, las condiciones en las que la educación superior de repente tuvo que realizar investigación y lo que ahora se suele denominar "educación en línea de emergencia"; los estudiantes necesitan asistencia; el personal se enfrenta a retos sin precedentes, incluida la inseguridad laboral; los dirigentes universitarios tuvieron que reinventar el funcionamiento de sus campus" (Marinoni et al., 2020, p. 6).

El Ministerio de Educación de Chile facilitó el uso de plataformas de enseñanza en línea para las instituciones que no disponían de estas herramientas, se abrieron fondos destinados al desarrollo y fortalecimiento de proyectos de educación en línea, se hicieron alianzas con distintas instituciones de educación superior para la difusión de buenas prácticas y para capacitar a docentes en modalidad en línea, se entregaron beneficios para estudiantiles de educación superior para facilitar su conectividad, y se solicitó a las distintas instituciones mantener información actualizada respecto al proceso educativo remoto. Así, del contexto de la crisis, surgió una nueva oportunidad de educación remota. En el contexto de este estudio, entenderemos a la educación como el desarrollo de programas de formación que tienen como escenario de enseñanza y aprendizaje el ciberespacio. Sin que se dé necesariamente un encuentro cara a cara entre el profesor y el alumno, es posible establecer una relación interpersonal de carácter educativo. Desde esta perspectiva, la educación virtual es una acción que busca 
propiciar espacios de formación, apoyándose en las TIC para instaurar una forma de enseñar y de aprender (Yong et al., 2017).

Finalmente, para contextualizar el estudio, es pertinente mencionar que la Educación Superior en Chile está conformada por tres tipos de instituciones reconocidas por el estado, tanto públicas como privadas: Universidades (59), Institutos Profesionales (39) y Centros de Formación Técnica (52), siendo la Universidad la que define el más alto nivel de enseñanza. En general, todas ellas cobran aranceles de ingreso, para los cuales se han diseñado apoyos o beneficios del estado para garantizar el acceso a personas de menores recursos (becas, créditos, gratuidad de arancel), para paliar en parte las brechas en el acceso propias de la situación socioeconómica de los estudiantes (Labraña y Vanderstraeten, 2020).

\section{OTROS ANTECEDENTES}

En un estudio sistemático de la literatura sobre trabajos científicos referidos a educación remota, se concluyó que no existen diferencias significativas entre los resultados obtenidos en los formatos presenciales y en la modalidad a distancia y en línea (Alonso-García et. al, 2019). Entonces, existiría la posibilidad de entregar una educación remota de alta calidad, con los resguardos instruccionales necesarios para garantizar procesos de enseñanza, aprendizaje y evaluación remota y, de esta forma, cumplir con lo comprometido en el formato presencial. A continuación, se presentan cuatro aspectos centrales que distintos autores han destacado para cumplir con una educación remota de calidad.

El primer elemento que aseguraría un proceso de calidad es considerar la competencia digital de los estudiantes y docentes para participar en la modalidad remota (Durán y Estay, 2016; García-Peñalvo y Corell, 2020a). Si bien es cierto que el uso de internet, de los recursos de la web y de las tecnologías de información se han convertido en un entorno habitual para el aprendizaje -ya que cuentan con sistemas ricos, dinámicos, interconectados, diversos y complejos, en los que se crea y se comparte el conocimiento (González-Sanmamed et al., 2018)_, no se puede asumir que todos estudiantes que participan del proceso tienen acceso a las últimas tecnologías o son expertos en su uso (Sun y Chen, 2016). Es más, existe poca evidencia para apoyar la afirmación de que la alfabetización digital, la conectividad, la necesidad de inmediatez, hacen a una generación de estudiantes, en particular, aprendices nativos o digitales (Gallardo-Echenique et al., 2015).

Los jóvenes pasan cada día más horas delante de los medios digitales, siendo grandes consumidores y productores de material de internet, sin embargo, la mayor parte de las veces este tiempo se usa en el uso recreacional de plataformas de redes sociales (Durkee, 2012). Los estudiantes llegan a la universidad con una cierta alfabetización digital, ya que conocen algunas herramientas TIC y las saben utilizar, pero siguen sin tener las competencias necesarias para aplicar esta alfabetización y el dominio de estas herramientas en un contexto educativo y, en concreto, en su proceso de formación para el aprendizaje (Henderson et al., 2015).

Los profesores, por otra parte, también son deficientes en estas habilidades, como lo expresan Koehler y Mishra (2013), ya que un importante número de profesores en ejercicio se formaron cuando los avances tecnologicos eran muy inferiores a los actuales, por tanto, no desarrollaron las competencias tecnológicas necesarias para los desafios de una educación virtual. Si a esto le sumamos la inversión de tiempo requerida para aprender estas habilidades y el necesario cambio de creencias pedagógicas, es una dificultad que no tiene rápida solución. Esta realidad se vuelve más compleja porque, como se sabe, enseñar no es solo transmitir información, sino que es problematizar la enseñanza en sí misma, descubrir y testear cómo hacer que los estudiantes aprendan de la forma más efectiva posible en su contexto. A estos desafíos se suma que la enseñanza virtual exige desarrollar y mejorar el trabajo pedagógico a través de la tecnología digital en un contexto profesional de enseñanza remota (From, 2017). Por lo tanto, el desarrollo de habilidades digitales en los profesores es un tema de preocupación. Las investigaciones han mostrado que la alfabetización digital es clave en toda disciplina y profesión, por lo que es necesario promoverla desde cualquier programa educativo (Durán y Estay, 2016). Es más, Koehler y Mishra (2013) ya habían planteado la necesidad de rediseñar el currículum para incorporar conocimiento tecnológico que permita a los docentes cubrir esta deficiencia.

Un segundo aspecto tiene que ver con la vinculación afectiva y pedagógica de los docentes con sus estudiantes. Uno de los puntos más criticados por los estudiantes que cursan estudios en línea es la falta de interacción social con sus compañeros (Mc Brien et al., 2009). La satisfacción de los estudiantes con una asignatura remota se relaciona con la percepción de "presencia social", es decir, que sientan que, independiente de la distancia física, existen medios para interactuar significativamente con sus docentes y, especialmente con sus compañeros, para construir y comprender los saberes (Sun y Chen, 2016), como también para colaborar entre ellos (Smith y Baik, 2019). En definitiva, recibir una buena educación no es la única razón por la que los estudiantes buscan ir a la 
universidad. Otras razones incluyen socializar, obtener experiencias de vida y hacer redes profesionales (Kaplan y Haenlein, 2016). La educación remota también debe considerar estos ámbitos.

Es común que los estudiantes se sientan más aislados y extrañen el contacto cara a cara en la educación remota. También es más fácil perder el hilo y el sentido con el estudio y las tareas asignadas, generándose barreras para comprometerse con actividades sincrónicas de aprendizaje (Owens et al., 2009). En este contexto, es aún más relevante la necesidad de una comunicación efectiva entre los estudiantes y los docentes para enfrentar estas adversidades (Smith y Baik, 2019). Según Akbar (2016), los programas de educación remota deben ser capaces de usar múltiples canales de comunicación con los estudiantes. Es importante asegurar un aprendizaje interactivo a través de foros de debate y blogs que posibiliten la discusión de temas relevantes para los universitarios, el desarrollo de proyectos de investigación, la resolución de problemas y el estudios de casos (García et al., 2015), como también usar tecnologías como iLectures, y eLive (Owens et al., 2009).

Se ha observado que el acceso a los servicios de asesoramiento, supervisión y tutoría en línea tiene ventajas en el compromiso y motivación de los estudiantes para participar en la enseñanza y el aprendizaje a distancia (Durán y Estay, 2016). Los estudiantes requieren directrices para planificar y ejecutar el estudio autónomo mediante la entrega de instrucciones claras y materiales de apoyo de buena calidad, elaborados o adaptados por el docente para la asignatura, además de acompañamiento y guía a través de retroalimentación formativa, para luego avanzar hacia actividades evaluadas con nota (Sun y Chen, 2016).

Un tercer aspecto destacado al que debe responder la educación remota tiene relación con la enseñanza activa y focalizada. Alonso-García et al. (2019) señalan que en la educación en línea es necesario que la asignatura esté claramente planificada, focalizada en los aspectos centrales y con una estructura de clase que permita hacer síntesis y cierres de lo aspectos más importante de cada sesión. La organización debe ser eficiente y con instrucciones claras, proporcionando a los estudiantes representaciones precisas de los conocimientos y conceptos enseñados. Por otra parte, se debe dejar en claro cuál es el objetivo de la clase y la relevancia de lo que están aprendiendo. En esta modalidad educativa, la docencia requiere ser intensiva y activa, utilizando estrategias de enseñanza constructivistas, donde el estudiante tiene un rol activo y no sólo receptivo. Las clases deben ser intelectualmente desafiantes y se les debe comunicar a los y las estudiantes expectativas positivas sobre su desempeño. Al utilizar distintos modos de presentar el conocimiento y permitir a los estudiantes que expresen su comprensión del tema de distintas formas, se respeta la diversidad de capacidades y modos de aprendizaje (Noben et al., 2020; Smith y Baik, 2019).

Finalmente, un aspecto muy relacionado con lo anterior tiene que ver con la evaluación auténtica del aprendizaje. Las evaluaciones siguen siendo el problema de fondo de la educación remota (García-Peñalvo y Corell, 2020a). Los docentes y los encargados de la gestión académica y curricular de las universidades están permanentemente buscando lograr evaluaciones equitativas entre las secciones de un mismo curso y buscando la manera de evitar la copia y comprometer a los estudiantes en su aprendizaje (Durán y Estay, 2016). Para Smith y Baik (2019), la respuesta tiene que ver con impulsar la innovación y facilitar oportunidades de aplicación del conocimiento en situaciones de práctica auténticas o simuladas, entregando a los estudiantes oportunidades para participar en el aprendizaje inductivo.

El sistema de evaluación, más que ser punitivo, debe permitir un aprendizaje más profundo, el desarrollo de nuevas ideas y permitir que el estudiante sea capaz de demostrar el dominio del contenido en torno al pensamiento crítico, la resolución de problemas, la colaboración y el aprendizaje autodirigido (Becker et al., 2017). Liberman et al., (2020) destacan la importancia de la evaluación formativa en contextos de aprendizaje remoto, especialmente en la pandemia COVID-19. Esto involucra continua retroalimentación sobre las tareas, lo que puede realizarse de manera sincrónica como asincrónica y con distintas plataformas y tecnologías, incluidos mensajes de audio por WhatsApp. Alonso-García et al. (2019) enfatiza la importancia de proporcionar retroalimentación inmediata, para que el estudiante pueda corregir y mejorar su desempeño. Asimismo, Harrison et al. (2020) sugieren implementar regularmente actividades de evaluación sumativa y formativa a través de revisión entre pares y la autoevaluación. Según la literatura especializada, estos cuatro aspectos son fundamentales para asegurar un proceso educativo remoto de calidad. Por lo tanto, resulta de interés indagar cómo se desarrollaron estos aspectos en este primer año de pandemia en Chile. El objetivo de esta investigación es caracterizar el proceso educativo remoto a nivel nacional durante la pandemia desde la experiencia de los docentes y estudiantes, considerando aspectos teóricos consensuados sobre las mejores prácticas de la educación remota y las diferencias por género y área disciplinar. 


\section{MÉTODO}

En este estudio se utiliza un diseño cuantitativo no experimental para analizar las percepciones de estudiantes y profesores sobre el proceso de enseñanza y aprendizaje remoto. El enfoque metodológico es de alcance transversal y de corte descriptivo-exploratorio, bajo la óptica de la estadística inferencial para establecer comparaciones entre grupos estudiantes-docentes, como también, entre categorías de estudiantes, como lo es el género y la disciplina. Para ello, el análisis utiliza pruebas t para muestras independientes, y análisis ANOVA. El procesamiento y análisis de los datos se realizó mediante el software SPSS® 22.

\section{Participantes}

En esta medición participaron 654 docentes (36\% hombres y $64 \%$ mujeres) y 1750 estudiantes (33\% hombres y $67 \%$ mujeres) pertenecientes a 34 universidades del país. El $44.9 \%$ de los estudiantes cursaba el ciclo inicial (primer y segundo año), el $42.1 \%$ el ciclo intermedio (tercer y cuarto año) y el $13 \%$ el ciclo terminal (quinto año en adelante). Se hizo un muestreo por conveniencia o accesibilidad, sin que los participantes hayan sido seleccionados mediante criterios estadísticos respecto de los informantes. Del conjunto de universidades, 11 de ellas eran públicas y 23 privadas, 21 de ellas con carácter regional y 13 con presencia en la capital nacional Santiago.

\section{Instrumentos}

De acuerdo al marco teórico analizado se diseñaron dos instrumentos de características similares para ser aplicados en modalidad en línea, uno de ellos orientado a estudiantes y otro a docentes. Los ítems de ambos instrumentos se diseñaron buscando recoger información respecto de cinco variables: a) condiciones físicastecnológicas y competencias digitales para educación remota, b) relación profesor- estudiantes, c) características del proceso de enseñanza en educación remota, d) características del proceso de evaluación en educación remota y e) emociones positivas y negativas en educación remota en pandemia. Además, para conocer diferencias por categoría de interés, género y área disciplinar, se indagó en 10 ámbitos comunes del proceso de consulta a estudiantes y docentes: empatía, didáctica, trabajo grupal, tutoría, autoevaluación, respeto, recursos, participación, aprendizaje y exigencia. Las áreas disciplinares se clasificaron en Artísticas, Ciencias Sociales, Salud, Educación, Ingenierías y Ciencias Biológicas. El cuestionario de los estudiantes consideró 65 preguntas y el de profesores 56. Ambos instrumentos consideraron ítems de respuesta cerrada, tipo Likert, de cinco puntos, de 1 (nunca) a 5 (siempre), además de cuatro preguntas abiertas para complementar aspectos cualitativos que pudieran potenciar la información. En cuanto a la consistencia interna, ambos cuestionarios exhiben características adecuadas, con un Alfa de Cronbach de 0.89 para el cuestionario de alumnos y 0.83 para el de profesores.

\section{Procedimiento}

Primeramente, se elaboró una versión preliminar de los cuestionarios, que fue revisada por pares externos para recibir retroalimentación en cuanto a comprensión, extensión y aspectos formales. Los cuestionarios fueron posteriormente presentados al comité de ética de la Universidad del Desarrollo, el cual validó su apego a las normativas éticas propias de estudios con seres humanos, de acuerdo a los objetivos de la investigación. Posteriormente, a través de redes académicas del equipo de investigadores asociados al proyecto, se generaron contactos con las 34 universidades para solicitar formalmente la colaboración para aplicar ambas encuestas. Estas encuestas estuvieron disponibles durante un periodo de 2 meses para ser respondidos en cualquier momento, de manera de no comprometer o perjudicar otras actividades académicas. Si bien el procedimiento de muestreo fue por conveniencia, el espectro público-privado de las universidades participantes permitió acceder a una gran amplitud y diversidad poblacional como para considerar una visión representativa de la educación superior remota en el contexto COVID-19 a nivel nacional.

\section{RESULTADOS}

A continuación, en un primer bloque, de manera descriptiva se informan las cinco categorías de las cuales se recogió información: a) condiciones físicas-tecnológicas y competencias digitales para educación remota, b) relación profesor-estudiantes, c) características del proceso de enseñanza en educación remota, d) características del proceso de evaluación en educación remota, y e) emociones positivas y negativas en educación remota en pandemia; un segundo bloque se exploran potenciales diferencias en las respuestas de acuerdo a categorías de interés. 


\section{Condiciones físicas-tecnológicas y competencias digitales}

A nivel global, el $87.7 \%$ de los estudiantes declararon contar con dispositivos electrónicos para sus clases en línea y el $79.5 \%$, tener conexión a internet. Sin embargo, casi el $40 \%$ de ellos indica no disponer de espacio físico adecuado para el trabajo académico. En el caso de los docentes, el $99 \%$ de ellos declara contar con dispositivos electrónicos y el $98.1 \%$ con conexión a internet, aunque más de un $20 \%$ de ellos indica no disponer de un espacio físico adecuado. Respecto a sus competencias para realizar educación remota, el $81.4 \%$ de los profesores universitarios declara contar con las competencias para realizar docencia en línea, pero más del 30\% de ellos considera que las capacitaciones recibidas desde sus casas de estudio no fueron totalmente útiles para enfrentar este desafío. Por otro lado, sólo el $13.4 \%$ de los estudiantes se sentían cómodos con activar su cámara en clases, aun cuando el $70 \%$ consideraba positivo que sus profesores lo hicieran.

\section{Relación profesor-estudiantes}

Más del $90 \%$ de los estudiantes consultados percibió respeto entre estudiantes y profesores en las clases en línea, lo que es igualmente corroborado por los docentes en un porcentaje similar. Más del $70 \%$ de los estudiantes indican que los docentes escuchan opiniones de los alumnos y muestran interés porque sus estudiantes aprendan en la modalidad remota. Sin embargo, existen diferencias en la percepción de estudiantes y docentes respecto al interés de estos últimos sobre la situación personal y familiar de éstos en el contexto de la pandemia. Así, sólo el $42.4 \%$ de los estudiantes indica que sus docentes mostraron preocupación por este tema, lo que contrasta con el hecho que más del $80 \%$ de los docentes señala haber preguntado cómo estaban ellos y sus familias con el confinamiento debido a la pandemia.

\section{Características del proceso de enseñanza en educación remota}

El $84.3 \%$ de los estudiantes señalan haber tenido sus clases en modalidad sincrónica, aunque casi el $40 \%$ de ellos considera que la cantidad de clases sincrónicas en cada semana era mayor a la que podían manejar. Respecto de las actividades pedagógicas habituales declaradas por los profesores, las más frecuentes fueron a) uso de powerpoint, envío de texto para leer antes de la clase, aplicación de guías, b) uso de ciertas tecnologías como videos cortos, podcast, pizarras zoom, padlet, youtube y c) enseñanza utilizando organizadores gráficos, mapas conceptuales o cuestionarios en línea. Dentro de las estrategias menos recurridas en esta etapa de educación en confinamiento, se relevaron con menos del $20 \%$ de ocurrencia: a) uso de herramientas lúdicas tipo kahoot y menti, y b) actividades de construcción colaborativa en línea.

\section{Características del proceso de evaluación en educación remota}

En relación al sistema de evaluación del aprendizaje, sólo el $42 \%$ de los estudiantes plantea haber recibido algún tipo de retroalimentación sobre su trabajo en modalidad remota. Por otra parte, un escaso $12 \%$ de los estudiantes muestra alta confianza en las evaluaciones recibidas. En cuanto a la honestidad académica, el 19\% de los alumnos reconoce haber copiado alguna vez y casi el $40 \%$ de los estudiantes cree que sus compañeros copiaron durante el semestre. Estos números están alineados con la opinión de los profesores, ya que solo el $26.2 \%$ de ellos plantea alta confianza en la evaluación, mientras que el $40.7 \%$ cree que sus alumnos copiaron. Más del $50 \%$ de los estudiantes relatan ser evaluados a través de tareas basadas en desempeño (como trabajos de investigación, ensayos, proyectos) y preguntas de respuesta abierta en las pruebas escritas. También es alto el porcentaje de estudiantes que informa que sus pruebas fueron de selección múltiple $(60.1 \%)$ e ítems de verdadero o falso $(30.2 \%)$.

\section{Emociones positivas y negativas en educación remota en pandemia}

Consultados respecto a las emociones que experimentaron durante la educación remota en el semestre pandemia, el $82 \%$ de los estudiantes relata haber sentido estrés, el $71 \%$ frustración y el $47 \%$ angustia. En todas estas emociones, las mujeres expresan mayor intensidad que los hombres. Las emociones negativas fueron más intensas en los estudiantes de primer y segundo año que en los pares de cursos superiores. Respecto a los docentes, el $73 \%$ de ellos relata haber sentido estrés, el 53\% frustración y el 35.5\% incertidumbre e inseguridad. Las profesoras mujeres informan significativamente mayor nivel de estrés y angustia que los hombres, mientras que los hombres informan más aburrimiento que las mujeres.

\section{Análisis comparativo de acuerdo a categorías de interés}

Es igualmente importante contrastar si existen diferencias en las percepciones declaradas en algunos ámbitos respecto de grupos específicos, tales como Empatía, Didáctica, Trabajo Grupal, Tutoría y Prácticas de Autoevaluación respecto de categorías de interés. Para tal efecto se contrastaron diferencias de medias entre género y disciplinas formativas en estudiantes y docentes. 


\section{Diferencias por género}

Respecto de la Empatía en pandemia —entendida como el interés de los docentes por conocer la situación de los estudiantes y sus familias en el contexto de la pandemia, preguntando y dedicando tiempo para conocer respecto de sus situaciones y atender opiniones de los estudiantes- se observa una mayor autopercepción de empatía en el caso de las profesoras $(M=4.211)$ respecto de sus pares varones $(M=3.950)$, siendo estas diferencias significativas $(\mathrm{t}=-3.336 ; P<.001)$. Por el lado de los estudiantes, de igual manera, las estudiantes mujeres tienden a percibir un grado de empatía superior de parte de sus docentes $(M=3.39)$ respecto de sus pares varones $(M=3.06)$, siendo esta diferencia significativa $(t=-7.334, P<.001)$. Se puede observar, en ambos casos, que la percepción de empatía de los docentes es levemente modulada por parte de los estudiantes.

También se indagó en diferencias respecto de la Didáctica, entendida como la preocupación y despliegue de recursos y mecanismos pedagógicos adecuados en pos de un entendimiento cabal de la asignatura, que transmita la sensación global de una clase bien preparada, organizada e impartida, considera preparar y disponer de material previo a la clase, establecer pausas o descansos durante la actividad, modulación y gesticulación del profesor y dominio de instancias de apertura, desarrollo y cierre de la clase. Dentro del grupo de profesores se observa una alta autopercepción del dominio de la didáctica en educación remota, con medias de apreciación de 4.48 para los profesores, y de 4.53 para las profesoras, diferencias que no resultan ser significativas ( $\mathrm{t}=-1.942$, $P=.053)$. Por el lado de los estudiantes, la apreciación de esta variable es mayor por las mujeres $(M=3.47)$ que por los hombres $(\mathrm{M}=3.24)$, siendo esta diferencia estadísticamente significativa $(\mathrm{t}=-6.564, \mathrm{P}<.001)$. Es decir, las estudiantes mujeres perciben una mejor didáctica en clases online que los hombres.

En cuanto al trabajo grupal, entendido como aquellas instancias que permiten a los docentes organizar trabajo entre pares, monitorear y percibir dicha modalidad como efectiva y agradable, igualmente se observan diferencias superiores en favor de la autopercepción de las docentes respecto de sus pares masculinos ( $M=3.74$ vs 3.39 ; $t=-$ $4,138, P<.001)$. Por el lado de los estudiantes, igualmente, el trabajo grupal es mejor percibido por las estudiantes mujeres $(\mathrm{M}=3.31)$ que sus pares masculinos $(\mathrm{M}=3.05 ; \mathrm{t}=-5.976, P<.001)$. Nuevamente, se puede observar que la percepción declarada sobre el trabajo grupal por parte de los docentes es levemente modulada por parte de los estudiantes.

Por el lado de las tutorías, entendidas como espacios de tiempo adicionales a las clases para resolver dudas o profundizar contenidos ya sea de manera individual o en grupos pequeños, no se observan diferencias significativas por género en la autopercepción de los docentes $(\mathrm{M}=3.18$ y $\mathrm{M}=3.28$ para profesores y profesoras respectivamente). En lo que respecta a los estudiantes, es también el ámbito con más baja evaluación, con medias de $M=2.15$ y $M=2.27$ para hombres y mujeres respectivamente, diferencias que no resultan ser estadísticamente significativas $(\mathrm{t}=-1.955, \mathrm{p}=.051)$, e igualmente con una diferencia del orden de un punto de apreciación en la escala respecto de la opinión de los docentes.

Finalmente, en el ámbito de prácticas de Autoevaluación instaladas por los docentes en las sesiones online entendido como aquellas instancias o momentos brindados en la clase para la autoevaluación, la evaluación a los compañeros y la percepción de aprendizaje al participar de estos mecanismos de evaluación-, los profesores declaran una escasa realización de este tipo de actividades, con medias de $M=2.23$ y $M=2.56$, para hombres y mujeres, las cuales resultan significativas $(\mathrm{t}=-3.623, P<.001)$. De igual forma, los estudiantes reportan la escasa realización de estas actividades en sus clases, con medias de $M=1.91$ y $M=2.22$ para hombres y mujeres, diferencias que resultan ser significativas $(\mathrm{t}=-6.603, P<.001)$. En ambos casos, las mujeres (estudiantes y docentes) perciben mayor frecuencia de estas actividades de autoevaluación en comparación a los estudiantes y docentes hombres.

\section{Diferencias por ámbito disciplinar}

Las carreras inscritas por el conjunto de estudiantes que participaron del proceso de consulta se clasificaron en las siguientes áreas disciplinares: Artísticas, Ciencias Sociales, Salud, Educación, Ingenierías y Ciencias Biológicas. Como cada disciplina puede tener sus propios énfasis metodológicos, es interesante analizar si los factores transversales anteriormente analizados son percibidos de manera diferente por parte de los estudiantes dependiendo del área de la carrera que estudian. Se realizó para ello un análisis ANOVA para comparar diferencias en diferentes aspectos. Adicionalmente a las cinco categorías anteriores; Empatía, Didáctica, Trabajo Grupal, Tutoría y Autoevaluación, se agregaron los siguientes cinco: Respeto, Recursos, Participación, Aprendizaje y Exigencia (Tabla 1). 
Tabla 1: ANOVA de 1 factor respecto de ámbitos asociados a la Educación En línea por disciplinas.

\begin{tabular}{|c|c|c|c|c|c|c|}
\hline \multicolumn{2}{|c|}{ Categorías } & Suma de cuadrados & $g l$ & Media cuadrática & $F$ & Sig. \\
\hline \multirow{3}{*}{ Empatía } & Entre grupos & 62,698 & 5 & 12,540 & 16,640 &, 000 \\
\hline & Dentro de grupos & 1302,211 & 1728 & ,754 & & \\
\hline & Total & 1364,909 & 1733 & & & \\
\hline \multirow{3}{*}{ Respeto } & Entre grupos & 1,352 & 5 & ,270 & ,957 &, 443 \\
\hline & Dentro de grupos & 487,961 & 1728 & ,282 & & \\
\hline & Total & 489,313 & 1733 & & & \\
\hline \multirow{3}{*}{ Recursos } & Entre grupos & 43,145 & 5 & 8,629 & 10,764 & 000 \\
\hline & Dentro de grupos & 1385,301 & 1728 & 802 & & \\
\hline & Total & 1428,446 & 1733 & & & \\
\hline \multirow{3}{*}{ Didáctica } & Entre grupos & 10,801 & 5 & 2,160 & 4,603 & 000 \\
\hline & Dentro de grupos & 811,023 & 1728 &, 469 & & \\
\hline & Total & 821,824 & 1733 & & & \\
\hline \multirow{3}{*}{ Participación } & Entre grupos & 10,620 & 5 & 2,124 & 3,502 & ,004 \\
\hline & Dentro de grupos & 1047,950 & 1728 & ,606 & & \\
\hline & Total & 1058,569 & 1733 & & & \\
\hline \multirow{3}{*}{$\begin{array}{l}\text { Trabajo } \\
\text { Grupal }\end{array}$} & Entre grupos & 34,324 & 5 & 6,865 & 9,385 &, 000 \\
\hline & Dentro de grupos & 1264,021 & 1728 & ,731 & & \\
\hline & Total & 1298,345 & 1733 & & & \\
\hline \multirow{3}{*}{ Tutoría } & Entre grupos & 58,399 & 5 & 11,680 & 9,068 & 000 \\
\hline & Dentro de grupos & 2225,665 & 1728 & 1,288 & & \\
\hline & Total & 2284,064 & 1733 & & & \\
\hline \multirow{3}{*}{ Aprendizaje } & Entre grupos & 5,171 & 5 & 1,034 & 1,577 & ,163 \\
\hline & Dentro de grupos & 1133,215 & 1728 & ,656 & & \\
\hline & Total & 1138,386 & 1733 & & & \\
\hline \multirow{3}{*}{ Autoevaluación } & Entre grupos & 134,601 & 5 & 26,920 & 31,030 &, 000 \\
\hline & Dentro de grupos & 1499,134 & 1728 & ,868 & & \\
\hline & Total & 1633,734 & 1733 & & & \\
\hline \multirow{3}{*}{ Exigencia } & Entre grupos & 3,743 & 5 & ,749 & 1,005 & ,413 \\
\hline & Dentro de grupos & 1287,812 & 1728 &, 745 & & \\
\hline & Total & 1291,555 & 1733 & & & \\
\hline
\end{tabular}

Del total de estos diez ámbitos, solo en tres de ellos, Respeto, Aprendizaje y Exigencia, no se encontraron diferencias significativas entre pares de grupos ( $F=.957, p=.443 ; F=1.577, p=.163 ; F=1.005, p=.413)$. El ámbito de Respeto se refiere a la percepción de respeto hacia el profesor por parte de la clase y desde el profesor hacia sus estudiantes. El ámbito de Aprendizaje se refiere a la percepción de la importancia de asistir y participar de la clase para lograr aprendizajes, de que se pueden lograr todos los aprendizajes esperados de la asignatura bajo la modalidad en línea y que se autoperciben las capacidades necesarias para lograr los aprendizajes de las asignaturas. El ámbito de Exigencia se refiere a la percepción del tiempo, esfuerzo y concentración que le significa participar de las clases en línea.

En el ámbito de Empatía, el análisis de comparaciones múltiples releva diferencias significativas entre las formaciones de Ingeniería respecto de Ciencias Sociales y Educación $(P<.001)$. En el ámbito de recursos, referidos a equipos, espacio físico adecuado en el hogar, y conexión a internet, las diferencias se observan entre Ciencias Sociales e Ingenierías $(P<.001)$, y también entre Ciencias Sociales y Salud, respecto de Educación $(P<.001)$. Respecto del ámbito de Didáctica, las diferencias se aprecian nuevamente entre las Ingenierías y las disciplinas de Ciencias Sociales $(P<.002)$, Salud $(P<.008)$ y Educación $(P<.018)$.

En lo referido a participación, entendida como una instancia dialógica para interactuar con los profesores, realizar preguntas, y tener espacios para intercambiar y escuchar opiniones de otros, se observan diferencias entre las Ingenierías y las carreras de Ciencias Sociales $(P<.012)$ y de Educación $(P<.002)$. En el ámbito de Trabajo Grupal, las diferencias residen principalmente entre las carreras Artísticas respecto de las de Ciencias Sociales $(P<.003)$, Salud $(P<.035)$ y Educación $(P<.001)$, como así también entre las Ingenierías y las Ciencias Sociales $(P<.001)$ y Educación $(P<.001)$. Respecto del ámbito de tutorías, se observan diferencias entre las carreras de Educación respecto de Ciencias Sociales $(P<.001)$, Salud $(P<.011)$ e Ingenierías $(P<.001)$. Finalmente, respecto del ámbito de Autoevaluación, se observan diferencias entre una mayor cantidad de grupos, como Educación respecto de Ciencias Sociales $(P<.001)$, Salud $(P<.002)$, Ingenierías $(P<.001)$ y Ciencias Biológicas $(P<.001)$, como, asimismo, entre las Ingenierías y las carreras Artísticas $(P<.012)$, Ciencias Sociales $(P<.001)$ y Salud $(P<.018)$. 


\section{DISCUSIÓN}

La educación remota tiene altas probabilidades de "llegar para quedarse". Por un lado, la alta capacidad de transmisión de COVID-19 sugiere que las medidas de confinamiento y distanciamiento social persistirán por un tiempo, y, por lo tanto, la educación remota deberá continuar como la opción formativa en la educación superior. Por otro lado, en el año 2020 se sentó un precedente disruptivo, mostrando que es posible llevar a cabo tanto el estudio como el trabajo desde el hogar. Probablemente, la crisis sanitaria continuará presionando por desarrollar y perfeccionar estas nuevas modalidades de trabajo remoto a fin de comprender mejor los alcances de las condiciones fisico-tecnológicas del hogar para la vida familiar, laboral y educativa. En particular, esta investigación buscó caracterizar el proceso educativo remoto a nivel nacional durante la pandemia desde la experiencia de los docentes y estudiantes, considerando aspectos teóricos consensuados sobre las mejores prácticas de la educación remota, y las diferencias por género y área disciplinar.

A partir de los hallazgos encontrados es necesario enfatizar un hecho evidente: la enseñanza presencial es distinta a la remota (Gordon et al., 2011). Curiosamente, a pesar de este hecho evidente, el análisis anterior muestra que ambas modalidades no necesariamente derivan en prácticas distintas, probablemente debido a la poca experiencia anterior a la pandemia que tenía la mayoría de los profesores en cuanto a la enseñanza en línea. Los profesores siguen utilizando las mismas estrategias e instrumentos utilizados en la enseñanza presencial. Como lo platean Noben et al. (2020), se deben buscar distintos modos de presentar y expresar el conocimiento, respetando la diversidad y el contexto, para instalar, de esta forma nuevos procedimientos, reconociendo las diferencias entre estas modalidades. En cuanto al perfeccionamiento, se infiere que los profesores no sólo requieren ser actualizados en aspectos tecnológicos sobre el uso de plataformas, sino también, como lo plantea From (2017), en la pedagogía que subyace a la educación remota y que difiere de una clase presencial.

Se confirma la necesidad, de acuerdo a Sun y Chen (2016), de favorecer la participación y el diálogo con y entre los estudiantes a través de preguntas abiertas y la problematización de los temas de la clase, y destinar un rol más protagónico a los estudiantes, por ejemplo, con exposiciones de parte de ellos. Asimismo, acorde con lo propuesto por Smith y Baik (2019), es importante perfeccionar el trabajo grupal en línea entre los estudiantes con un monitoreo y retroalimentación por parte del docente sobre el trabajo que se está realizando. En este sentido, es relevante promover el contacto entre los estudiantes y el sentido de pertenencia a un curso y una carrera, porque es lo que más extrañan los estudiantes y es lo que los contiene emocionalmente. La articulación de las necesidades socioemocionales y educativas se funden de manera efectiva en una docencia bajo un enfoque constructivista, que active e invite al diálogo de los estudiantes, en donde la activación de conocimientos previos inicie con espacios de escucha cotidiana como una buena manera de empatizar y contender las experiencias informales o cotidianas, para luego vincularlas a los procesos de aprendizaje que se trabajan en las instancias sincrónicas.

Por otro lado, la educación en línea es distinta a la educación remota en emergencia. No sólo ha cambiado la modalidad de educación de presencial a remota, sino que también el contexto, esto es: en pandemia, con distanciamiento social y cuarentena. Como lo han confirmado diversos estudios (Nearchou et al., 2020), en este contexto la salud mental se ve afectada y, tanto los docentes como los estudiantes, no están en las mismas condiciones cognitivas y afectivas para abordar los desafíos de la virtualidad. Se observa en un porcentaje mayoritario, tanto en estudiantes como en docentes, experimentación de emociones negativas como el estrés y la frustración. Por estos motivos, es necesario cuidar y mantener un vínculo positivo entre docentes y estudiantes, destinando minutos de la clase para saber cómo están ellos, sus familias y cómo están sobrellevando este tiempo. Esto es especialmente importante con los estudiantes de los primeros años de universidad, que tienden a sentirse más invadidos de emociones negativas. Los resultados muestran, igualmente, importantes diferencias de género respecto al tipo de emoción negativa predominante.

Se confirma lo dicho por García-Peñalvo y Corell (2020a) en cuanto a que la evaluación sigue siendo el aspecto más deficitario en la educación remota. Ciertamente, la modalidad en línea puede verse como un riesgo inherente para que surjan actitudes o prácticas contrarias a la integridad académica, como se ve reflejado por las aprehensiones de los propios estudiantes y profesores respecto de la percepción de copias en las pruebas. Sin embargo, también es necesario recordar el alto porcentaje pruebas declaradas en modalidad de selección múltiple e ítems de verdadero o falso, en donde resulta más fácil compartir resultados de manera rápida. Por lo mismo, como lo plantean Smith y Baik (2019), es recomendable llevar a cabo actividades más significativas y sustantivas, favoreciendo la construcción e involucramiento de los estudiantes con el conocimiento y diseñando tareas innovadoras de desempeño grupal e individual, que resulten desafiantes cognitivamente y que fomenten la aplicación y uso del saber para tomar decisiones cada vez más profesionales. De la misma manera, se pueden 
aprovechar los temas contingentes, situaciones de la vida cotidiana y problemas típicos de la profesión para traerlos a la clase. Incorporar alguna una evaluación individual y oral durante el semestre también permite mayor conocimiento de los estudiantes y aumenta la confianza en la evaluación de parte de los profesores y estudiantes. Estos últimos aspectos, buscan remirar la evaluación desde una perspectiva de diseño instruccional acorde a la educación en línea; esto quiere decir, centrar la atención en la secuencia de contenidos, actividades y recursos, así como considerar la ética digital como elementos claves para el diseño de la evaluación desde la perspectiva del docente que propone y del estudiante que es evaluado (García-Peñalvo, 2020b). Un aporte sustancial a esta transformación, podría ser la propuesta desde las Universidades Españolas; quienes han propuesto bajo una metodología descriptiva y diversa, alternativas importantes para adaptar la evaluación en el contexto del COVID19 (Grupo de trabajo intersectorial de CRUE Universidades Españolas, 2020).

Asimismo, es importante hacer más retroalimentación y menos calificación. En tiempos de pandemia, en coherencia con lo planteado por Alonso-García et al. (2019), se requiere involucrar de manera más significativa al estudiante en su proceso de mejora y avance, lo que es más factible de hacer a través de reuniones individuales o en grupos pequeños, entregando retroalimentación sobre sus trabajos; dando la oportunidad de corregir y mejorar sus productos, y no sólo explicando la puntuación asignada. Estas prácticas de retroalimentación, que informan sobre lo logrado, lo que falta por lograr y cómo alcanzar el desempeño deseado, favorecen la autorregulación y motivación de los estudiantes. Posicionar la retroalimentación como un espacio dialógico y longitudinal del proceso formativo, permite no sólo analizar los resultados y las demostraciones que ellos logran; sino que igualmente, favorece la auto-observación como un mecanismo que permita guiarlos en espacios de construcción de conocimientos carentes, todavía, de presencialidad y contacto social.

Respecto del género, si bien existen diferencias importantes en algunas dimensiones a favor de las mujeres (una percepción más positiva de la presencia de la empatía, trabajo grupal y autoevaluación), tanto en el grupo de docentes como el de estudiantes (en este grupo también está presente la didáctica a favor de las mujeres), el hallazgo relevante en términos generales, pareciera ser que la autopercepción de los docentes es significativamente mayor que aquella percibida por los estudiantes en los mismos ámbitos de comparación. Esto puede estar destacando, por una parte, un esfuerzo importante por parte de los docentes para ejercer la docencia con dedicación, pero con ciertas falencias percibidas por los estudiantes. Estas pueden deberse a múltiples factores, tales como, por ejemplo, la aún incipiente experiencia en metodologías en línea, la calibración de tiempos y espacios adecuados para el trabajo académico y estudio de parte de los estudiantes.

En relación a las áreas de conocimiento, se ven diferencias recurrentes entre las formaciones de Ingenierías, Educación y Ciencias Sociales. Si bien hay aspectos que pueden ser modulados por las propias disciplinas, el que existan diferencias significativas en el ámbito de los recursos disponibles puede estar evidenciando un aspecto de segmentación socioeconómica respecto de formaciones universitarias que debe ser atendido al momento de planificar la enseñanza remota y las políticas institucionales. En general, en cuanto a disposición de equipos, las diferencias son mínimas, lo que puede explicarse por las políticas de cobertura en equipos y chips de conectividad que en general implementaron rápidamente las universidades hacia sus estudiantes. Sin embargo, los aspectos de conectividad y espacios físicos tienen que ver con características asociadas al hogar o lugar de residencia principal, ya que, como consecuencia de la pandemia, muy pocos estudiantes declararon otros espacios para conectarse o realizar trabajo académico fuera del hogar. De hecho, en las preguntas de respuesta abierta, varios de ellos declararon haberse visto en la necesidad de trabajar para apoyar el presupuesto familiar, o bien, de no contar con espacios para poder llevar a cabo un trabajo académico con cierta tranquilidad, ya sea por la cantidad de integrantes del núcleo familiar o por estar al cuidado de hijos o sobrinos. Por último, es relevante plantear que una limitante del trabajo es su característica transversal, que impide ver la evolución adaptativa de una serie de elementos que es esperable que vayan experimentando cambios al mantenerse la modalidad remota, al menos por un semestre académico adicional.

Hasta el momento, a pesar de la existencia de las vacunas y su efecto preventivo, no se visualiza un plazo determinado para poder dar por superada la pandemia. Más aun, tampoco se tiene mayor conocimiento acerca de cómo serán las dinámicas formativas en educación superior con posterioridad a ésta. Lo cierto es que transcurridos ya tres semestres con modalidad de educación remota, hay una serie de aspectos locales y externos, que deberán repensarse para poder mantener la consistencia de la formación: movilidad interinstitucional, prácticas o pasantías, trabajo en terreno, talleres o laboratorios, desarrollo de habilidades, y otras particularidades propias de cada formación que están a la base del propio prestigio de cada carrera de pregrado. El conocimiento de las percepciones de estudiantes y docentes respecto a su interacción en la enseñanza remota puede contribuir a la búsqueda de soluciones a estos problemas, al permitir anticipar la disposición que tendrán frente a estos desafíos. 
Como limitaciones del trabajo podemos mencionar en primer lugar, el hecho que la encuesta se aplicó una única vez, no permitiendo por lo tanto, capturar la evolución de las percepciones de los estudiantes y profesores debido a que la modalidad remota se mantiene al menos hasta el primer semestre 2021. Esto cobra especial interés, toda vez que los profesores van adquiriendo mayor experiencia, y además, van siendo retroalimentados por los estudiantes en las tradicionales evaluaciones a la calidad de la docencia que suelen realizar las universidades al término de cada semestre, lo que podría estar incubando un proceso de adaptación, o bien, al mantenerse o agravarse, estar en presencia de una expectativa de abandono de esta modalidad apenas las condiciones lo permitan. Otra limitación es la técnica de muestreo utilizada (no probabilístico y no aleatorio), que impide el poder inferir algunas de estas conclusiones como fielmente representativas de la educación superior chilena. Así las perspectivas en las cuales se trabaja actualmente tienen que ver con realizar nuevas aplicaciones de la encuesta para analizar esta evolución, y ampliar la muestra para poder llevar a cabo otro tipo de análisis, como modelos de ecuaciones estructurales que muestren dinámicas de interacción conjunta entre los distintos factores estudiados respecto de otras variables de dominio cognitivo.

\section{CONCLUSIONES}

De acuerdo a los resultados expuestos se pueden extraer las siguiente conclusiones: a) las prácticas docentes de enseñanza-aprendizaje siguen siendo similares para presencialidad y virtualidad, necesitándose no solo formación tecnológica sino también formación pedagógica para introducir cambios a nivel de enseñanza y evaluación en la educación online; b) se hace necesario en este contexto (virtualidad y pandemia) favorecer la participación y el diálogo con los estudiantes y entre ellos (individuales y grupales), no solo para una pertinente retroalimentación del proceso formativo, sino también para conocer lo que sucede alrededor de dicho proceso. Dicha acción se hace expecialmente relevante en los primeros años de Universidad, de forma de cuidar y mantener un vínculo positivo entre docentes y estudiantes, y para favorecer la autorregulación y motivación de estos últimos; c) la evaluación debe mejorarse de manera significativa, no solo para monitorear los aprendizajes, sino también para fortalecer las confianzas entre los estudiantes y docentes. Se debe innovar, en este ámbito, en acciones más significativas y sustantivas que eviten la copia y promuevan aprendizajes de calidad; d) la autopercepción de los docentes es significativamente más positiva que la de los estudiantes en los mismos ámbitos de comparación, posiblemente por la responsabilidad y liderazgo que implica el proceso; e) existen diferencias importantes por género, en donde las mujeres, tanto en estudiantes como en profesores, perciben mayor presencia de la empatía, el trabajo grupal y la autoevaluación, que los hombres; f) existen diferencias especialmente entre las formaciones de Ingenierías, Educación y Ciencias Sociales, aunque parte de las causas pueden ser moduladas por las propias disciplinas, todo indica que las diferencias socioeconómicas puede ser un factor de importancia.

\section{REFERENCIAS}

Alonso-García, S., Aznar-Díaz, I., y otros tres autores, Systematic review of good teaching practices with ICT in Spanish higher education. Trends and challenges for sustainability, https://doi.org/10.3390/su11247150, Sustainability, 11(24), 1-15 (2019)

Akbar, M., Digital technology shaping teaching practices in higher education, https://doi.org/10.3389/fict.2016.00001, Frontiers in ICT, 3(1), 1-5 (2016)

Becker, S. A., Cummins, M. y otros cuatro autores, NMC horizon report: 2017 higher education edition, The New Media, Austin, Texas, USA (2017)

Durán R., y Estay, C., Formación en buenas prácticas docentes para la educación virtual, https://doi.org/10.5944/ried.19.1.13845, RIED, 19(1), 209-232 (2016)

Durkee, T., Prevalence of pathological internet use among adolescents in Europe: demographic and social factors, http://dx.doi.org/10.1111/j.1360-0443.2012.03946.x, Addiction, 107(12), 2210-2222 (2012)

From, J., Pedagogical digital competence-between values, knowledge and skills, https://doi.org/10.5539/hes.v7n2p43, HES. Higher Education Studies, 7(2), 43-50 (2017)

Gallardo-Echenique, E., Marqués-Molías, L., Bullen, M., y Strijbos, J., Let's talk about digital learners in the digital era, https://doi.org/10.19173/irrodl.v16i3.2196, The International Review of Research in Open and Distributed Learning (IRRODL), 16(3), 156-187 (2015)

García-Peñalvo, F. J., Modelo de referencia para la enseñanza no presencial en universidades presenciales, Campus Virtuales, 9(1), 41-56 (2020)

García-Peñalvo, F. J., y Corell, A., La COVID-19: ¿Enzima de la transformación digital de la docencia o reflejo de una crisis metodológica y competencial en la educación superior?, Campus Virtuales, 9(2), 83-98 (2020) 
García, A., Proenza, G., Segundo, R., y Granados, J. M., Buenas prácticas en los entornos virtuales de enseñanzaaprendizaje, Revista Cubana de Educación Superior, 34(3), 76-88 (2015)

González-Sanmamed, M., Sangrà, A., Souto-Seijo, A., y Estévez, I., Ecologías de aprendizaje en la era digital: desafíos para la educación superior, https://doi.org/10.30827/publicaciones.v48i1.7329, Publicaciones, 48(1), 11-38 (2018)

Gordon, G., Sorensen, C., y otros cuatro autores, Overcoming student resistance to group work: online versus face-to-face, https://doi.org/10.1016/j.iheduc.2010.09.005, Internet High. Educ., 14, 121-128 (2011)

Grupo de Trabajo intersectorial CRUE Universidades Españolas, Informe sobre procedimientos de evaluación no presencial, Estudio del impacto de su implementación en las Universidades Españolas y recomendaciones, https://tic.crue.org/ (2020)

Harrison, R., Meyer, L., y otros cinco autores, Evaluating and enhancing quality in higher education teaching practice: a meta-review, https://doi.org/10.1080/03075079.2020.1730315, Stud. High. Educ., (2020)

Henderson, M., Selwyn, N., y Aston, R., What works and why? student perceptions of 'useful' digital technology in university teaching and learning, http://dx.doi.org/10.1080/03075079.2015.1007946, Stud. High. Educ., 42(8), 1567-1579 (2015)

Kaplan, A., y Haenlein, M., Higher education and the digital revolution: about MOOCs, SPOCs, social media, and the cookie monster, https://doi.org/10.1016/j.bushor.2016.03.008, Bus Horiz, 59, 441-450 (2016)

Koehler, M., y Mishra, P., What is technological pedagogical content knowledge (TPACK)?, https://doi.org/10.1177/002205741319300303, Journal of Education, 193(3), 13-19 (2013)

Labraña, J., y Vanderstraeten, R., Functional differentiation and university expansion in Chile, http://dx.doi.org/10.17583/hse.2020.4565, HSE - Social and Education History, 9(3), 252-277 (2020)

Liberman, J., Levin, V., y Luna-Bazaldua, D., Are students still learning during COVID-19? formative assessment can provide the answer, World Bank Blogs (2020)

López-Meneses, E., Gómez-Galán, J., Bernal-Bravo, C., y Vázquez-Cano, E., Fortalezas y debilidades de los cursos masivos abiertos en línea (MOOC) frente a otros modelos de enseñanza en contextos socio-educativos, http://dx.doi.org/10.4067/S0718-50062020000600077, Form. Univ., 13(6), 77-87 (2020)

Manyika, J., Lund, S., y otros cuatro autores, Digital globalization: the new era of global flows, Global Institute, San Francisco, USA (2016)

Marinoni, G., van't Land, H., y Jensen, T., The impact of Covid-19 on higher education around the world. IAU Global Survey Report. International Association of Universities (IAU) Global Survey Report. https://www.iau- aiu.net/ (2020)

Mc Brien, J.L., Jones, P., y Cheng, R., Virtual spaces: employing a synchronous on-line classroom to facilitate student engagement in online learning, International Review of Research in Open and Distance Learning, 10(3), 1- 7 (2009)

Nearchou, F., Flinn, C., y otros tres autores, Exploring the impact of COVID-19 on mental health outcomes in children and adolescents: a systematic review, https://doi.org/10.3390/ijerph17228479, Int. J. Environ. Res. Public Health, 17(22), 8479 (2020)

Noben, I., Folkert, J., y Hofman, W. H., Quality of teaching in higher education: reviewing teaching behaviour through classroom observations, https://doi.org/10.1080/1360144X.2020.1830776, Int. J. Acad. Dev., (2020)

Owens, J., Hardcastle, L., y Richardson, B., Learning from a distance: the experience of remote students, Journal of Distance Education, 23(3), 53-74 (2009)

Servicio de Información de Educación Superior (SIES), Informe matrícula 2019 en educación superior en Chile. Subsecretaría de Educación Superior, (2019)

Smith, C., y Baik, Ch., High-impact teaching practices in higher education: a best evidence review, https://doi.org/10.1080/03075079.2019.1698539, Stud. High. Educ., (2019)

Sun, A., y Chen, X., Online education and its effective practice: a research review, https://doi.org/10.28945/3502, Journal of Information Technology Education: Research, 15, 157-190 (2016)

Yong, E., Nagles, N., Mejía, C., y Chaparro, C., Evolución de la educación superior a distancia: desafíos y oportunidades para su gestión, Revista Virtual Universidad Católica del Norte, 50, 80-105 (2017) 